\title{
UNIQUENESS SETS FOR FOURIER SERIES
}

\section{ASHOT VAGHARSHAKYAN}

Abstract. This article explores some of the sets of uniqueness for Fourier series. The case, when these sets have zero Lebesgue measure is considered.

Mathematics subject classification (2010): 42A63.

Keywords and phrases: Fourier series; Fourier coefficients; uniqueness sets; exceptional sets.

\section{REFERENCES}

[1] N. BARI, Trigonometric series, Moscow (Russian), 1961.

[2] A. Broman, On two classes of trigonometrical series, Thesis, University of Uppsala, Uppsala, 1947.

[3] L. CARleson, Selected Problems on Exceptional Sets, Princeton University Press, New Jersey, 1967.

[4] A. VAGHARSHAKYAN, A theorem on the representation of harmonic functions, (Russian) Izv. Nats. Akad. Nauk Armenii Mat. 28 (1993), no. 4, 87-91 (1995); translation in J. Contemp. Math. Anal. 28 (1993), no. 4, 76-79.

[5] A. Vagharshakyan, On the uniqueness problem for Fourier series, Real Analysis Exchanges vol. 29 (2), 2003/2004.

[6] J.-P. Kahane, Series de Fourier absolument covergentes, Springer-Verlag, Berlin-Heidelberg-New York, 1970.

[7] A. ZyGmund, Contribution a l'unisite du developpement trigonometrique, MZ, 24 (1926). 